\title{
Mutations and Expression Profile of EDIL3 and Correlation with HIF1A and Tumor-Associated Carbonic Anhydrases in Pancreatic Cancer
}

\author{
Dilara Fatma Akin Bali ${ }^{1}$, Rahsan llikci Sagkan² $^{\mathbb{C}}$ \\ ${ }^{1}$ Nigde Omer Halisdemir University, Faculty of Medicine, Medical Biology, Nigde, Turkey \\ ${ }^{2}$ Usak University, Faculty of Medicine, Medical Biology, Usak, Turkey \\ Correspondence Author: Rahşan llikci Sagkan \\ E-mail: rahsan.ilikci@usak.edu.tr \\ Received: $23.06 .2020 \quad$ Accepted: 29.06.2021
}

\begin{abstract}
Objective: Epidermal growth factor-like repeats and discoidin I-like domains 3 (EDIL3) expression is upregulated in some types of cancer which means that it can be used as a candidate tumor marker. The expression level of EDIL3 as a marker of the hypoxic microenvironment of pancreatic cancer was assessed by studying its relationship with the expression of tumor-associated carbonic anhydrases (CA IX and CA XII) and Hypoxiainducible factor-1 (HIF-1) in tumor development.

Methods: Gene expression and mutation profiles of pancreatic cancer patients and healthy tissue samples were downloaded The Cancer Genome Atlas (TCGA), and the genetic alterations and expression levels of the EDIL3, HIF-1 $\alpha$, CA IX and CA XII genes were analyzed. Additionally, PolyPhen-2 and SNAP tools were used to prediction and confirmation of detected alterations pathogenicity and survival analysis was performed.

Results: Expression levels of EDIL3, HIF-1 $\alpha$ and CA IX were found to be statistically significant higher in the patient compared to healthy group and we showed also positive correlation between EDIL3 and HIF-1 $\alpha$ gene expression. Furthermore, low CA IX and CA XII expression levels were found effective on overall survival $(p<0.05)$. Additionally, 8 missense mutations were detected in the coding region of studied genes.

Conclusion: We suggested that relationship between EDIL3 and HIF-1 $\alpha$ in pancreatic cancer and EDIL3 is a novel molecule cancer development in pancreatic cancer.
\end{abstract}

Keywords: CAs, EDIL3, HIF-1 $\alpha$, mutation, expression, pancreatic cancer

\section{INTRODUCTION}

Pancreatic cancer, which has become increasingly frequent in recent years and known as the fourth fatal cancer, is a disease with an aggressive character which develops relatively asymptomatically and usually appears advanced in diagnosis (1). Additionally, the genetic and phenotypic heterogeneity of pancreatic cancers is a significant clinical problem. Understanding the interactions between the molecular mechanisms underlying pancreatic cancer progression will provide a significant improvement in the development of more selective and effective treatment models for medical therapy for pancreatic cancer $(2,3)$. The presence of hypoxic areas in the tumor microenvironment causes more unstable genetic structure, aggressive/ invasive phenotype, increase tumor volume, trigger epithelial mesenchymal transmission and escape of cancer cells from the immune system $(4,5)$. In addition, it causes resistance to alternative cancer treatment and a significant decrease in survival rates. Although the underlying mechanisms mediating pancreatic cancer invasion and metastasis are largely unknown, hypoxic microenvironment and hypoxia-related factors have been reported to be critical driving forces in pancreatic cancer metastasis and progression.

Hypoxia-inducible factor-1 (HIF-1) is the main effector in hypoxia activation by generation of the blood vessels, lymphatic vessels, fibroblasts, immune cells, and various extracellular matrix components $(6,7)$. The interaction of these input molecules with tumor cells cause to clinical outcomes. Most solid tumors have hypoxic regions because of abnormal vascularization and low blood supply. This character of hypoxia promotes numerous changes favor cancer progression (4-8). The expression of HIF-1 $\alpha$ gene activates signaling pathways related with cell metabolism and supports to glycolytic mode by producing $\mathrm{H}^{+}$. Furthermore, the activity of carbonic anhydrases (CA) is affected by HIF-1 $\alpha$ signalization. Zinc 
containing carbonic anhydrase enzymes have important roles in different physiological functions as gas exchange, ion transport and acid-base balance (10). There are 15 enzymatically active CA isoforms in mammalian and the role of these enzymes, participate in $\mathrm{pH}$ regulation, are catalysis of the hydration of $\mathrm{CO}_{2}$ molecule and dehydration of $\mathrm{HCO}^{3}$ - ion $(10,11)$. The molecular and cellular structure and functions of several carbonic anhydrases have been characterized. CA IX and CA XII, the major types of tumor associated proteins $(11,12)$. The discovery of transmembrane carbonic anhydrases which are CA IX and CA XII is a hallmark of cancer, especially angiogenesis, tumor invasion, progression and metastasis (10-12). Several studies have shown that cancer-related CA IX and CA X II expression can take place in cancer. These enzymes were identified in numerous cancer types such as human renal cancer, pancreatic, colon, prostate, ovaries, testicles, lung, brain, normal endometrial cells and epididymis duct cells (10-13). The association between malignant transformation and the expression of carbonic anhydrases could not be accentuated until the discovery of CA IX and CA XII from tumor-associated CA isozymes.

Epidermal growth factor-like repeats and discoidin I-like domains 3 (EDIL3 is also called DEL-1) is a secreted and multifunctional extracellular matrix protein that plays an important role in regulating cellular growth, differentiation, adhesion, and apoptosis in many biological systems especially tumor cells (14). EDIL3 has also an anti-inflammatory function because of inhibiting the recruitment of immune cells to inflammatory site. In addition, inflammation is critical in the progression of various types of carcinoma, and donates invasive and metastatic characteristics upon transformed cells $(15,16)$. The tumor microenvironment facilitates tumor metastasis to other organs. Various signaling pathways contribute to this abnormal process. The role of EDIL3 in cancer is complex and paradoxical, varying by cell type and stage of tumorigenesis (17). In the present bioinformatical study was done to determine the relationship between transmembrane carbonic anhydrases and EDIL3 mutation profile and m-RNA expression patterns in the data of pancreatic cancer and healthy samples. The function of EDIL3, HIF-1 accentuated and tumor-associated carbonic anhydrases are poorly understood. In this study, we have used bioinformatics tools to decipher the mutation profiles and expression levels of EDIL3, HIF-1 $\alpha$, CA IX and CA XII genes.

\section{METHODS}

\subsection{Data collection}

The pancreatic cancer data set was obtained from TCGA database. Demographic, clinical and genetic data regarding the patient group are summarized in Table 1.
Table 1. Demographic, clinical and genetic data of patients with pancreatic cancer

\begin{tabular}{|l|l|}
\hline Characteristic & Patient data n:640 (\%) \\
\hline Gender & \\
\hline $\begin{array}{l}\text { Male } \\
\text { Female }\end{array}$ & $340(53.1 \%)$ \\
NA & $281(43.9 \%)$ \\
\hline Diagnosis age, years & $19(3.0 \%)$ \\
\hline Race Category & 61 (range, 33-90) \\
\hline White & \\
\hline Black or African American & $161(25.2 \%)$ \\
\hline Asian & $7(1.1 \%)$ \\
\hline NA & $11(1.7 \%)$ \\
\hline Sample Type & $461(72 \%)$ \\
\hline Primary & \\
\hline Metastasis & $560(87.5 \%)$ \\
\hline NA & $3(0.5 \%)$ \\
\hline Overall Survival Status & $77(12 \%)$ \\
\hline Living & \\
\hline Deceased & $99(15.5 \%)$ \\
\hline NA & $85(13.3 \%)$ \\
\hline Metastasis Stage Code & $456(71.3 \%)$ \\
\hline MO & \\
\hline MX & $84(13.1 \%)$ \\
\hline M1 & $95(14.8 \%)$ \\
\hline NA & $5(0.8 \%)$ \\
\hline Tumor stage code & $456(71.3 \%)$ \\
\hline T1 & \\
\hline T2 & $7(1.1 \%)$ \\
\hline T3 & $23(9.1 \%)$ \\
\hline T4 & $148(23.1 \%)$ \\
\hline TX & $4(0.6 \%)$ \\
\hline NA & $1(0.2 \%)$ \\
\hline Alteration Frequency in pancreatic cancer & Case (Frequency\%) \\
\hline HIF-1A Mutation & $3(0.5 \%)$ \\
\hline EDIL3 Mutation & $2(0.4 \%)$ \\
\hline CAIX Mutation & $13(2.3 \%)$ \\
\hline CAXII Mutation & $1(0.2 \%)$ \\
\hline & \\
\hline
\end{tabular}

\subsection{Mutation Analysis}

The cBio Cancer Genomics Portal (http://cbioportal.org) is an open accessed, The Cancer Genome Atlas (TCGA)-based resource that allows interactive research of multidimensional cancer genomic datasets and provides access to the data of more than 5,000 tumor samples from 20 cancer studies $(18,19)$. Pancreatic cancer was chosen as the type of cancer of interest on the web interface to examine mutations in HIF-1 $\alpha$, EDIL3, CA IX and CA XII genes in pancreatic cancer patients presented in the portal. The selected TCGA data set consists of the genome sequencing data of 640 pancreatic cancer patients. The characteristics of the mutations detected are comprehensively analyzed using the OncoPrint and Mutation tools provided by the interface. 


\subsection{In-Silico Analysis}

To determine the possible pathogenicity of the mutations which we detected in our study genes; HIF-1 $\alpha$, EDIL3, CA IX and CA XII, we used the scores given by Polymorphism Phenotyping v2 (PolyPhen-2), Scanning of unacceptable polymorphisms (SNAP) and the Catalog of Somatic Mutations in Cancer (COSMIC) databases.

PolyPhen-2, which can be accessed via a web server, helps predict the possible effects of possible mutations on the stability and function of human proteins using the structural and comparative evolutionary analyses of the amino acid positions of these possible mutations. The program estimates the probability of the missense mutation being damaging based on a combination of all these features and provides both a qualitative prediction (probably damaging, possibly damaging, benign or unknown) with a score (20).

SNAP is a machine learning device called "neural network". It distinguishes between effect and neutral variants/nonsynonymous SNPs by taking a variety of sequence and variant features into account. It includes evolutionary constraints, structural features and protein annotation information. The most important single feature for SNAP prediction is conservation in a family of related proteins as reflected by PSIC scores (21). In the SNAP software, when the given values are between $-100-0$ and 0-100, the mutations are considered neutral and affected, respectively. Finally, the score given by the COSMIC database was used to predict and confirm the pathogenic effect of detected changes (22). Moreover, the evolutionary conservation analyses of the detected mutant amino acids were evaluated among different species via the "Multiple sequence alignment" tool in the PolyPhen-2 software.

\subsection{Gene Expression, Survival and Correlation Analysis}

GEPIA (http://gepia.cancer-pku.cn/) is an online database that contains the expression profiles of 9736 tumor samples and 8587 healthy samples. It is an interactive web server developed to provide customizable analyses such as differential expression analysis in tumor or normal tissues, profiling according to cancer types or pathological stages, patient survival analysis, similar gene detection, correlation analysis, and dimensionality reduction (23). The profiles of HIF-1A, EDIL3, CA IX, and CA XII gene expressions were analyzed as box plot graphs created by the GEPIA database using data from 179 pancreatic cancer patients and 171 healthy tissue samples obtained from TCGA and GTEx data. Furthermore, the correlation analyses between the expression levels of the EDIL3 gene and other study genes were done using the software. The $p$-values were automatically calculated by the software in both analyzes, and p-values below 0.05 were considered statistically significant. The survival analyses of the study genes according to their varying gene expression levels were done via the web interface.

\subsection{Statistical Analysis}

All statistical analyzes were carried out on the GEPIA database. Kaplan-Meier curves regarding overall survival. Low and high expression groups were compared used the log-rank test. Pearson test was performed for correlation analyses using online database. $\mathrm{p}<0.05$ was considered to indicate a statistically significant difference.

\section{RESULTS}

\subsection{Results of mutation analysis}

To identify the genetic changes in HIF-1 $\alpha$, EDIL3, CAIX, and CA $\mathrm{XII}$ genes in pancreatic cancer samples, genome sequencing data from 640 pancreatic cancer patients were analyzed via the cBioPortal interface. At least one genetic change (missense mutation) was detected in the HIF-1 $\alpha$, EDIL3, CA IX, and CA XII genes in $3.4 \%$ of all pancreatic cancer patients (Figure-1). Eight missense mutations (HIF-1A p.G429R, p.S692Y, p.N827D; EDIL3 p.L9I, p.D52Y; CAIX p.L293V, p.S294C; CA XII p.R240Q) were detected in all study genes. The changes detected in the studied genes as a result of the mutation analysis are shown in Table-2. CA IX was found to be the most frequently changed gene with a rate of $2.3 \%$ among the analyzed genes.

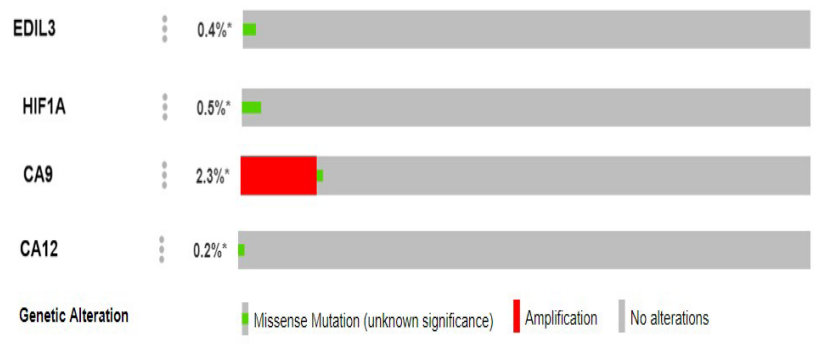

Figure 1. Distribution of mutations in EDIL3, HIF-1A, CA IX and CA XII genes in patients with pancreatic cancer

HIF-1 $\alpha$ has two transactivation domains (TAD) as the $\mathrm{NH}_{2}$ terminal (N-TAD) and the $\mathrm{COOH}$ terminal (C-TAD). These two domains are responsible for the transcriptional activity of HIF-1 $\alpha$. The C-TAD domain (between amino acids 786-826) interacts with coactivators such as CBP/ p300 to modulate the gene transcription of HIF-1 $\alpha$ under hypoxia. The p.N827D change detected in HIF-1 $\alpha$ in our study was located on the C-TAD domain. The oxygendependent degradation domain (ODDD) rich in Pro-SerThr amino acids is localized between amino acids 401-603 in the central region of HIF-1A, and it is responsible for the oxygen-dependent degradation of HIF-1 1 . The p.G429R detected in our study was located on this domain. The p.L293V and p.S294C missense mutations detected in the CA IX gene and the P.R240Q missense mutation detected in the CA XII gene were localized on the carbonic anhydrase active sites in both genes. 
Table 2. Mutations of the EDIL3, HIF-1A, CA IX and CA XII genes in pancreatic cancer patients.

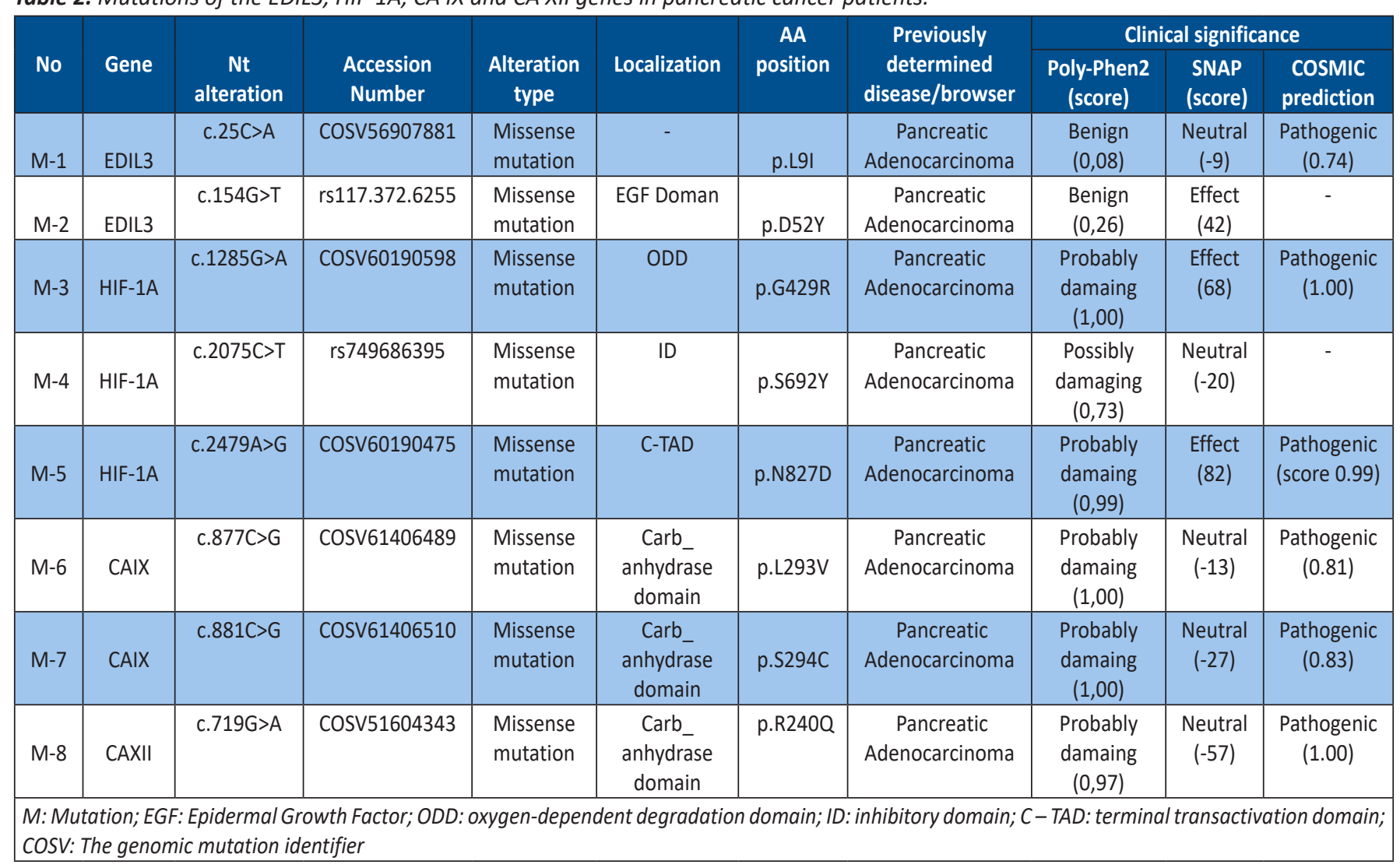

Moreover, 6 of the missense mutations mentioned above were recorded as somatic mutations in the COSMIC database. The schematic representation of the domain architecture of the proteins and mutations found in pancreatic cancer is shown in Figure 2.

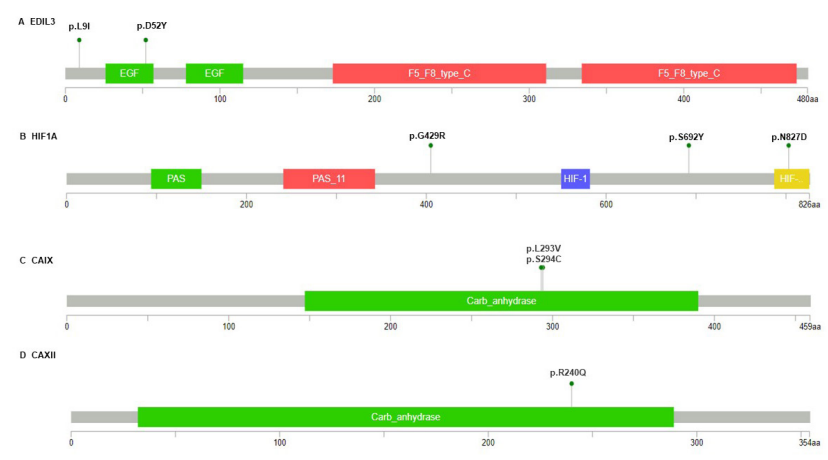

Figure 2. Schematic representation of domain architecture of the EDIL3, HIF-1A, CA IX and CA XII proteins and mutations found in pancreatic cancer patients. (A) Human EDIL3 is a polypeptide of 480 amino acids. (B) Human HIF-1A is a polypeptide of 826 amino acids (C) Human CA IX is a polypeptide of 459 amino acids. (D) Human CA $X I I$ is a polypeptide of 354 amino acids.

\subsection{Results of in-silico Analysis}

According to Poly-Phen2 database analysis results, 6 of the 8 missense mutations that were detected in the study genes and that are given in detail in Table-1 (HIF-1 $\alpha$ p.G429R, p.S692Y, p.N827D; CA IX p.L293V, p.S294C; CA XII p.R240Q) had a pathogenic score close to 1 , suggesting that they might have pathogenic characteristics.
The estimations for the pathogenic characteristics using the PolyPhen2 software are given in detail in Figure-3 (a-d).

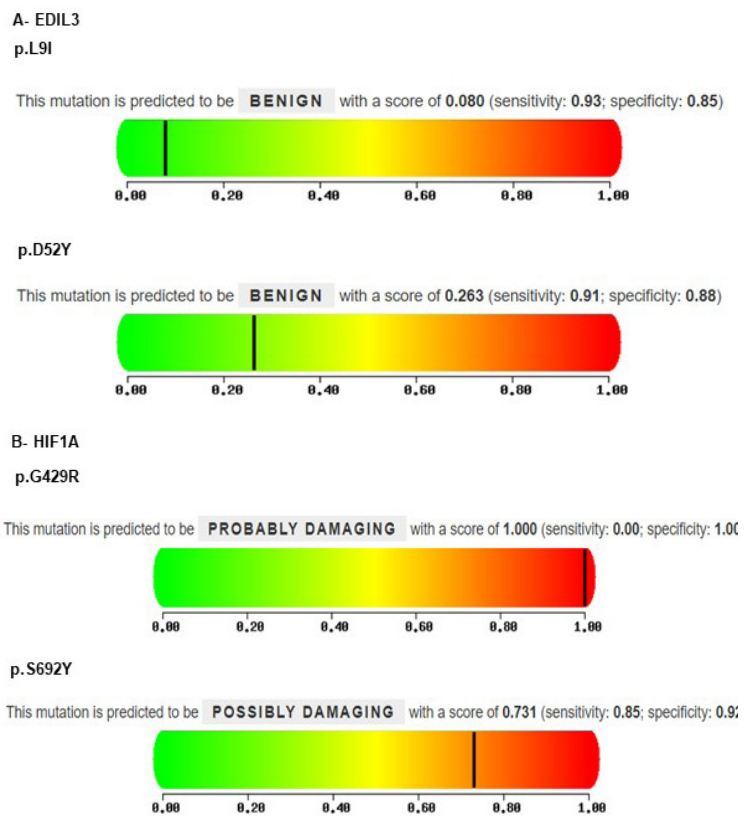

p.N803D

This mutation is predicted to be PROBABLY DAMA GING with a score of 0.999 (sensitivity: 0.14 ; specificity: 0.9

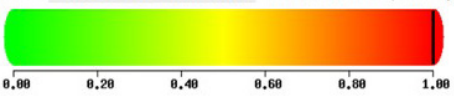




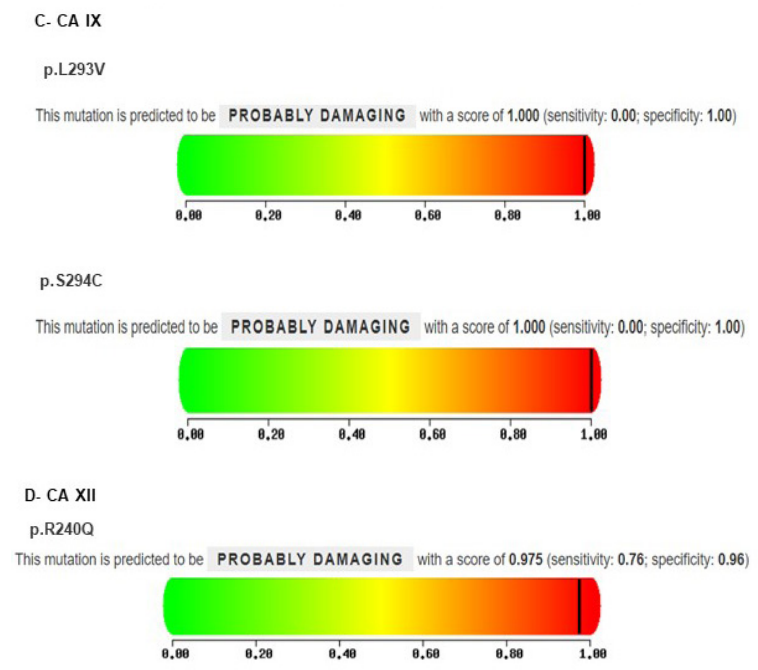

Figure 3. Estimation of possible functional effects of mutations in EDIL3, HIF-1A, CA IX and CA XII genes with PolyPhen-2 program.

In addition, the comparison of the amino acid sequences affected by the missense mutations detected in different species using the "Multiple sequence alignment" option in the Poly-Phen2 software showed that the p.G429R, p.S692Y, and p.N827D missense mutations detected in HIF-1 $\alpha$, the p.L293V, and p.S294C missense mutations detected in CA IX and the p.R240Q missense mutation detected in CA XII replaced their amino acids at critical points, which have been conserved among different species throughout their evolutionary process (Figure-4).

\subsection{Results of Gene Expression, Survival and Correlation Analysis}

Gene expression analysis was done to determine whether the gene expression profiles of HIF-1 $\alpha$, EDIL3, CA IX, and CA XII genes showed variation between pancreatic cancer patients and healthy samples. According to our results, the expression levels of HIF-1 $\alpha$, EDIL3, and CA IX were significantly higher in pancreatic cancer patients compared to normal tissues. CA XII was found to be lower in pancreatic cancer patients compared to the healthy group. The gene expression profile analyses for each gene were found to be statistically significant between the groups. In addition, the Pearson correlation between HIF-1 $\alpha$, CA IX, and CA XII mRNA expression results and EDIL3 expression levels was evaluated separately, and a positive correlation with a low correlation coefficient was determined between HIF-1 $\alpha$ and EDIL3 expressions. No correlation was found between CA IX, CA XII, and EDIL3 expressions (Figure 5). Additionally, in Figure 6, according to the log-rank test and $p$-value, the low expression levels of CA IX and CA XII were found effective on overall survival $(p=0.009$ and $p=0.000056$ respectively).
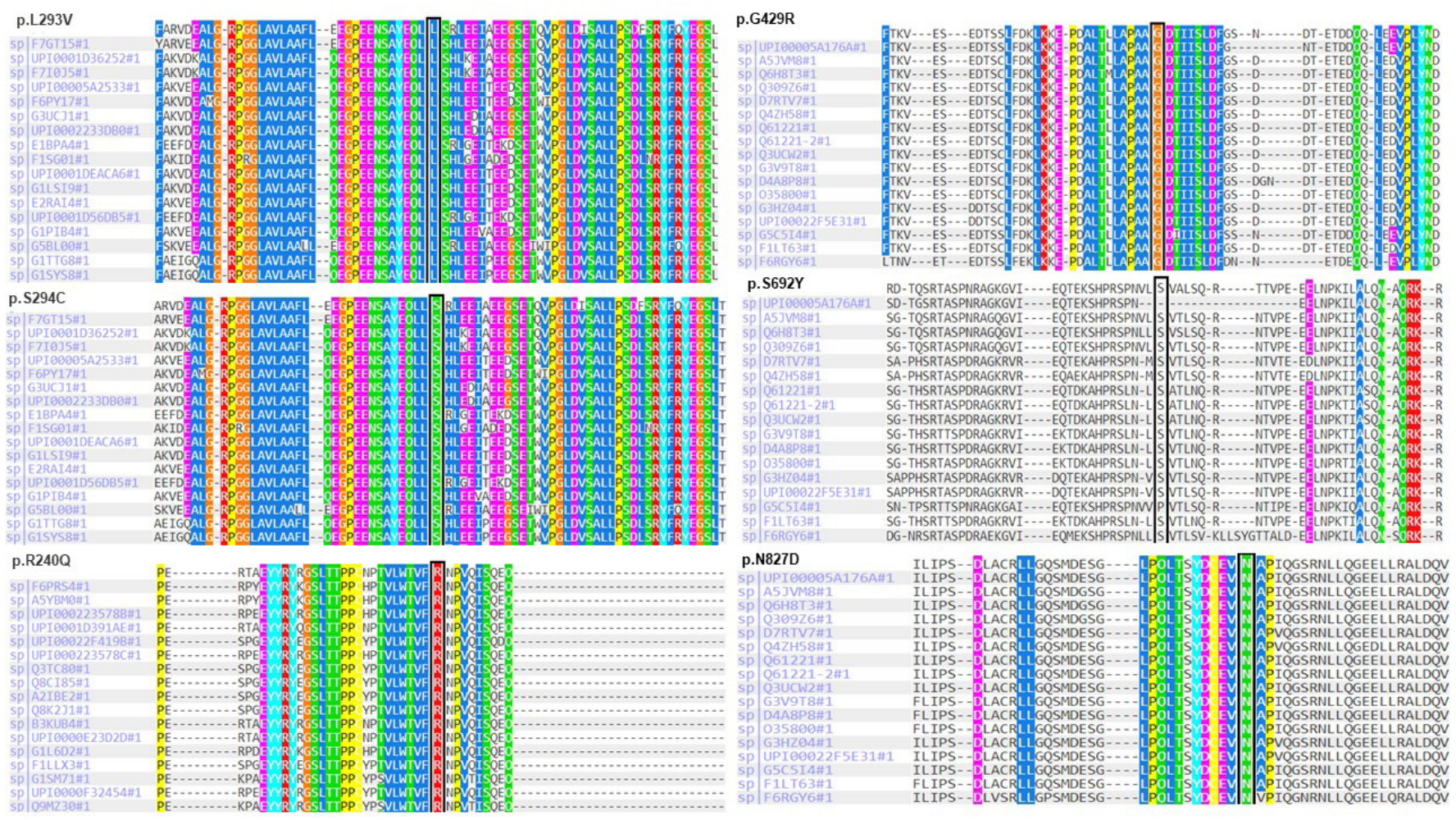

Figure 4. The evolutionary conservation analyses of the mutated amino acids in HIF-1A, CA IX and CA XII genes in the present study. The mutated amino acids were demonstrated. The detected mutant amino acids were evaluated among different species. 


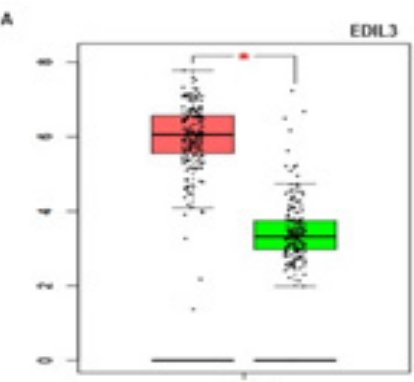

s

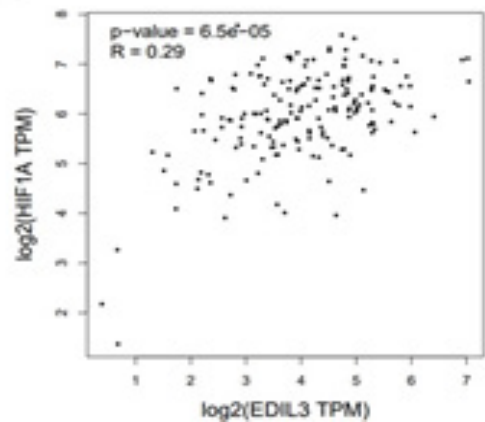

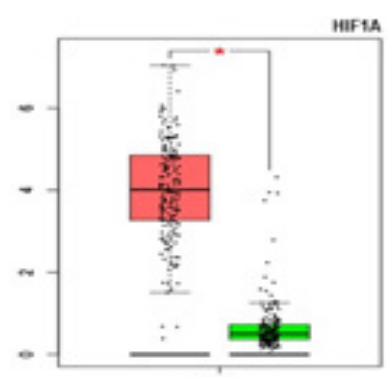

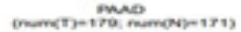

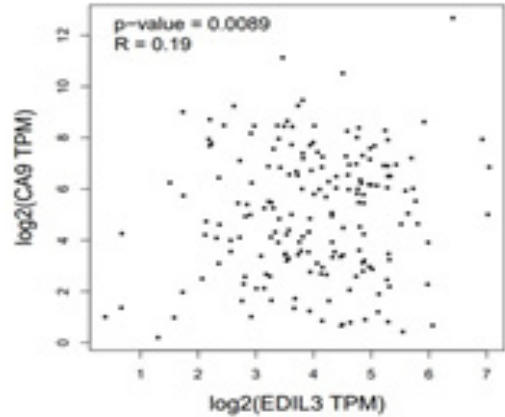

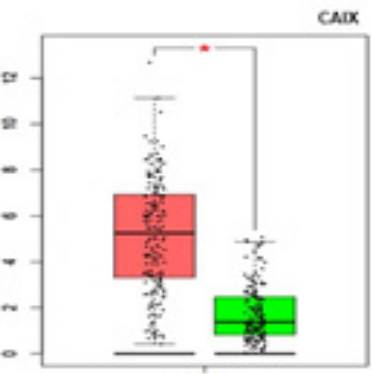

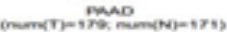

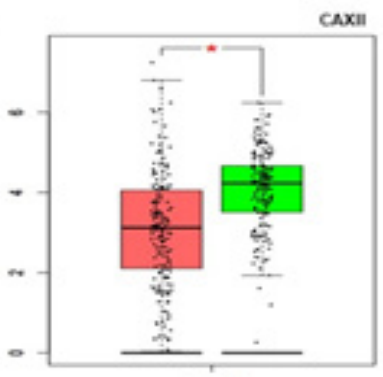

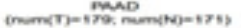

Figure 5. Validation of the m-RNA expression levels of (A) EDIL3, HIF-1A, CA IX and CA XII in pancreatic cancer tissues and normal pancreas tissues using GEPIA. These four box plots are based on 179 pancreatic cancer samples (marked in red) and 171 normal samples (marked in green). (B) Pearson correlation analyses of HIF-1A, CA IX and CA XII m-RNA expressions with EDIL-3 expression. ( ${ }^{*}$ indicates $\left.p<0.05\right)$.
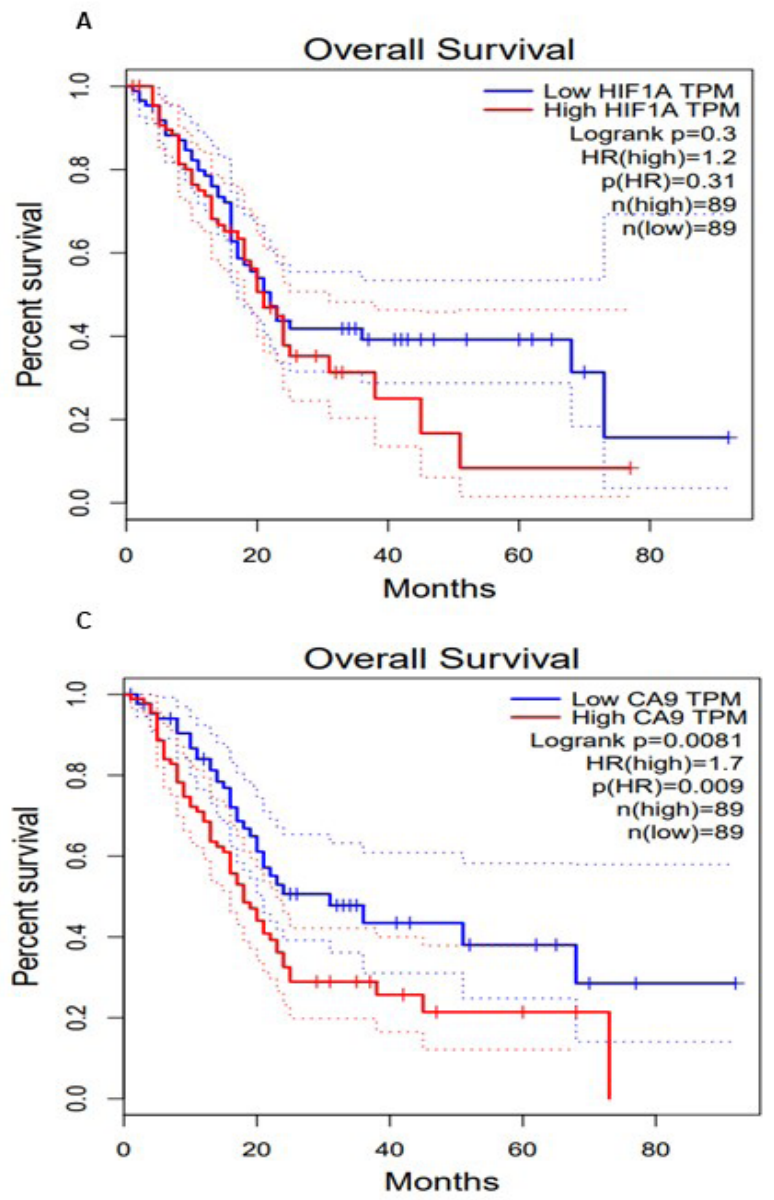
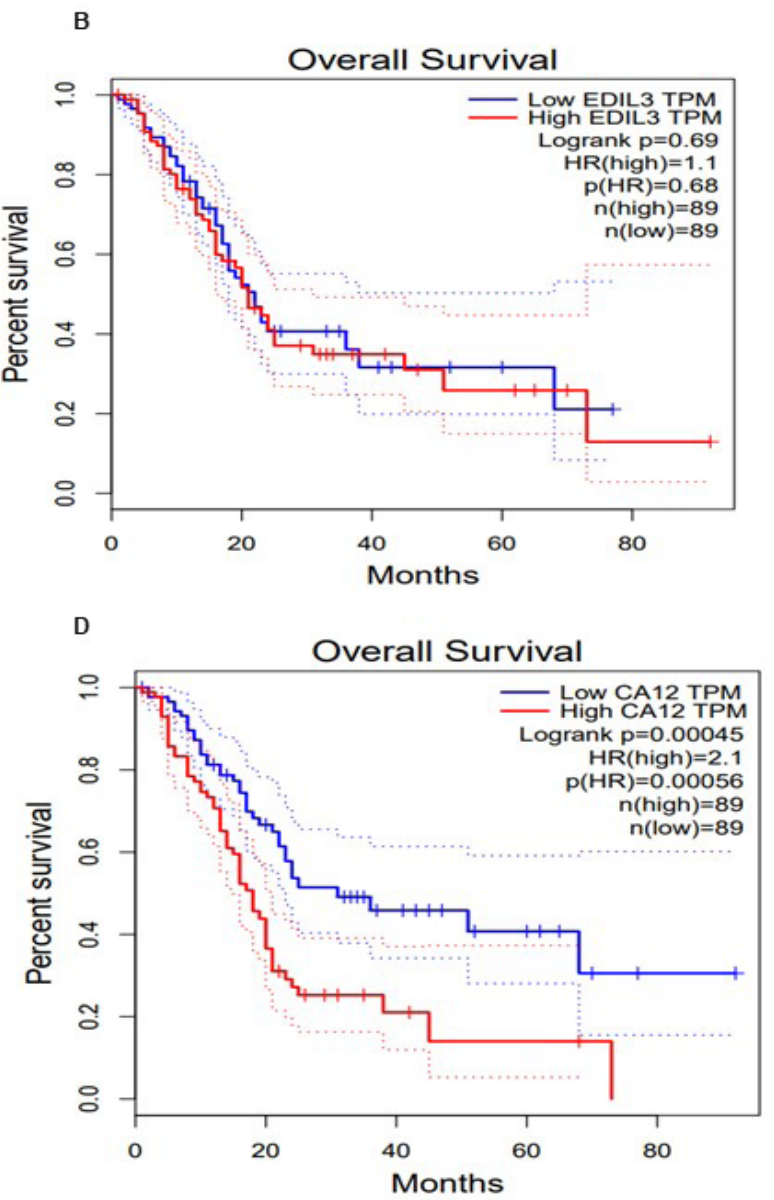

Figure 6. Kaplan-Meier analysis of overall survival and disease free survival of the 179 pancreatic patients according to different HIF-1A (A), EDIL3 (B), CA IX (C) and CA XII (D) levels. (*indicates $p<0.05)$. 


\section{DISCUSSION}

The adaptation of hypoxic tumor environment related to tumor progression and hypoxic nature is a unique characteristic in advanced pancreatic cancer like other solid tumors (24). The measurement of cellular responses to hypoxia may be clinical correlation with prognosis and prediction in treatment. HIF-1 $\alpha$ is a good hypoxia marker and also transcription factor $(5-7,24)$. Activation of HIF-1 $\alpha$ signal protein may lead to cell proliferation, prevention of programmed cell death and accelerating of tumor progression $(24,25)$. Carbonic anhydrase IX is one of the target gene of HIF- $1 \alpha$ and is known to play an important in angiogenesis, abnormal cell invasion and metastasis. Previous studies demonstrated that the induction of CA IX by HIF-1 in various cancer under hypoxic conditions (9-12,24,25). The association of HIF-1 $\alpha$, CA IX and EDIL3 expression has not yet been assessed in pancreatic cancer. In the present study, a significant association among CA IX, HIF-1 $\alpha$ and EDIL3 expression was reported in pancreatic cancer patients. Furthermore, we observed that CA IX and EDIL3 expressions were markedly increased at m-RNA level, accompanied by increased level of HIF-1 $\alpha$ in pancreatic cancer patients compared to healthy subjects. HIF-1 $\alpha$ regulates tumor angiogenesis and its overexpression has been associated with tumor angiogenesis as in our study group. It has been also reported that high HIF-1 $\alpha$ expression has a poor effect on the overall survival of pancreatic patients $(26,27)$. CAIX, which is a membrane-bound protein regulated by HIF-1 $\alpha$ under hypoxic conditions, also has a very high expression level in the pancreatic patient group, which includes our study group, compared to the healthy group. Interestingly, a positive correlation was identified between CA IX and HIF-1 $\alpha$ expression. Therefore, CA IX expression induced by hypoxia appears to critical role in tumor development by induction of neovascularization and enhancing inflammation relevance to EDIL3 expression. Considering the prognostic significance of EDIL3 expression status may be more reliable marker for predicting tumor aggressiveness associated with tumor hypoxia. Besides, patients with low CA IX and CA XII expression levels were found to have longer survival times than those with higher expression levels.

To determine the mutation profiles of EDIL3, HIF-1 $\alpha$, CA IX and CA XII genes, which are among the genome sequencing results of 640 pancreatic cancer patients in TCGA datasets, were comprehensively analyzed. We found that $3.4 \%$ of the patient group had a mutation and that the most frequent genetic changes occurred in CA IX (2.3\%). Missense mutations were identified in the study genes, especially in the sequences that encoded their important domains. According to the evolutionary analyses of the missense mutations we detected (HIF-1 $\alpha$ p.G429R, p.S692Y, p.N827D; CAIX p.L293V, p.S294C; CA XII p.R240Q ), we think that the mutations may have led to the formation of HIF-1 $\alpha$, CAIX, and CA XII genes with impaired functions and the genes were likely to have potential pathogenic characteristics since the functional pathogenic effects analyses showed that the mutations affected the amino acids that have been preserved throughout the evolutionary processes of these genes. Because these missense mutation points were locations that were highly conserved among species in the evolutionary process. Moreover, the missense mutations detected in all four genes were listed as somatic mutations in the COSMIC database. As there are no studies in the literature indicating the mutation profiles of the EDIL3, HIF-1 $\alpha$, CA IX, and CA XII genes and the role of the association between expression patterns in the pathogenesis of pancreatic cancer, our study is the first study stating the cancer-guiding roles of the mutations and expression profiles. Additionally, a further large-scale wetlab study is needed to evaluate the roles of EDIL3, HIF-1 $\alpha$, CA IX, and CA XII genes in pancreatic cancer.

\section{CONCLUSION}

The key findings of this in-silico analysis are that the overexpression of CA IX is induced by HIF-1 $\alpha$ in pancreatic cancer and also high level of CA IX expression are predictor of clinical outcome and decrease survival but no the correlation of EDIL3 and cancer-associated carbonic anhydrases. On the contrary, the relationship between EDIL3 and hypoxic marker HIF- $1 \alpha$ was found. In addition, the high expression level of EDIL3 might be good predictive and prognostic marker and as a novel research target related with tumor hypoxia in pancreatic cancer.

\section{Conflict of interest}

Authors declare no scientific and financial interest.

\section{Acknowledgements}

The data used in our study are obtained from public database the TCGA Research Network: https:// www.cancer.gov/tcga. We thank the TCGA and GEPIA databases for the availability of the data.

\section{Disclosure statement}

No potential conflict of interest was reported by the author(s).

\section{Funding}

No funding was received.

\section{Ethical approval and ethical standards}

The data used in our study were obtained from public database TCGA, therefore, ethical approval was not required.

\section{Availability of data and materials}

The datasets generated and analyzed during the current study are available in TGCA database (https:// www.cancer. gov/tcga), The cbio cancer genomics portal (http://www. cbioportal.org/). 


\section{REFERENCES}

[1] Rawla P, Sunkara T, Gaduputi V. Epidemiology of Pancreatic Cancer: Global Trends, Etiology and Risk Factors. World J Oncol 2019;10:10-27.

[2] Juiz NA, lovanna J, Dusetti N. Pancreatic Cancer Heterogeneity Can Be Explained Beyond the Genome. Front Oncol 2019;9:246.

[3] Makohon-Moore A, lacobuzio-Donahue CA. Pancreatic cancer biology and genetics from an evolutionary perspective. Nat Rev Cancer 2016;16:553-65.

[4] Araos J, Sleeman JP, Garvalov BK. The role of hypoxic signalling in metastasis: towards translating knowledge of basic biology into novel anti-tumour strategies. Clin Exp Metastasis 2018;35:563-599.

[5] Parks SK, Cormerais Y, Pouysségur J. Hypoxia and cellular metabolism in tumour pathophysiology. J Physiol 2017;595:2439-2450.

[6] Palazon A, Goldrath AW, Nizet V, Johnson RS. HIF transcription factors, inflammation, and immunity. Immunity 2014;41:51828.

[7] Petrova V, Annicchiarico-Petruzzelli M, Melino G, Amelio I. The hypoxic tumour microenvironment. Oncogenesis 2018;7:10.

[8] Al Tameemi W, Dale TP, Al-Jumaily RMK, Forsyth NR. HypoxiaModified Cancer Cell Metabolism. Front Cell Dev Biol 2019 29;7:4.

[9] Dengler VL, Galbraith M, Espinosa JM. Transcriptional regulation by hypoxia inducible factors. Crit Rev Biochem Mol Biol 2014;49:1-15.

[10] Mboge MY, Mahon BP, McKenna R, Frost SC. Carbonic Anhydrases: Role in pH Control and Cancer. Metabolites 2018; 28;8(1). pii: E19.

[11] Waheed A, Sly WS. Carbonic anhydrase XII functions in health and disease. Gene 2017 5;623:33-40.

[12] Pastorekova S, Gillies RJ. The role of carbonic anhydrase IX in cancer development: links to hypoxia, acidosis, and beyond. Cancer Metastasis Rev. 2019;38(1-2):65-77.

[13] Supuran CT. Carbonic anhydrase inhibitors as emerging agents for the treatment and imaging of hypoxic tumors. Expert Opin Investig Drugs 2018;27:963-970.

[14] Jiang SH, Wang $Y$, Yang JY, Li J, Feng $M X$, Wang YH, Yang XM, He P, Tian GA, Zhang XX, Li Q, Cao XY, Huo YM, Yang MW, Fu $X L$, Li J, Liu DJ, Dai M, Wen SY, Gu JR, Hong J, Hua R, Zhang ZG, Sun YW. Overexpressed EDIL3 predicts poor prognosis and promotes anchorage-independent tumor growth in human pancreatic cancer. Oncotarget 2016;7(4):4226-40.

[15] Choi EY, Chavakis E, Czabanka MA, Langer HF, Fraemohs L, Economopoulou M, Kundu RK, Orlandi A, Zheng YY, Prieto DA, Ballantyne CM, Constant SL, Aird WC, Papayannopoulou T, Gahmberg CG, Udey MC, Vajkoczy P, Quertermous T, Dimmeler S, Weber C, Chavakis T. Del-1, an endogenous leukocyte-endothelial adhesion inhibitor, limits inflammatory cell recruitment. Science 2008;322(5904):1101-4.

[16] Kourtzelis I, Li X, Mitroulis I, Grosser D, Kajikawa T, Wang B, Grzybek M, von Renesse J, Czogalla A, Troullinaki M, Ferreira A, Doreth C, Ruppova K, Chen LS, Hosur K, Lim JH, Chung KJ, Grossklaus S, Tausche AK, Joosten LAB, Moutsopoulos NM, Wielockx B, Castrillo A, Korostoff JM, Coskun Ü, Hajishengallis
G, Chavakis T. DEL-1 promotes macrophage efferocytosis and clearance of inflammation. Nat Immunol 2019;20(1):40-49.

[17] Jiang SH, Wang Y, Yang JY, Li J, Feng MX, Wang YH, Yang XM, He P, Tian GA, Zhang XX, Li Q, Cao XY, Huo YM, Yang MW, Fu $X L$, Li J, Liu DJ, Dai M, Wen SY, Gu JR, Hong J, Hua R, Zhang ZG, Sun YW. Overexpressed EDIL3 predicts poor prognosis and promotes anchorage-independent tumor growth in human pancreatic cancer. Oncotarget 2016;7(4):4226-40.

[18] Cerami E, Gao J, Dogrusoz U, Gross BE, Sumer SO, Aksoy BA, Jacobsen A, Byrne CJ, Heuer ML, Larsson E, Antipin Y, Reva B, Goldberg AP, Sander C, Schultz N. The cBio cancer genomics portal: an open platform for exploring multidimensional cancer genomics data. Cancer Discov 2012;2(5):401-4.

[19] Sanchez-Vega F, Mina M, Armenia J, Chatila WK, Luna A, La KC, Dimitriadoy S, Liu DL, Kantheti HS, Saghafinia S, Chakravarty D, Daian F, Gao Q, Bailey MH, Liang WW, Foltz SM, Shmulevich I, Ding L, Heins Z, Ochoa A, Gross B, Gao J, Zhang H, Kundra R, Kandoth C, Bahceci I, Dervishi L, Dogrusoz U, Zhou W, Shen H, Laird PW, Way GP, Greene CS, Liang H, Xiao Y, Wang C, lavarone A, Berger AH, Bivona TG, Lazar AJ, Hammer GD, Giordano T, Kwong LN, McArthur G, Huang C, Tward AD, Frederick MJ, McCormick F, Meyerson M; Cancer Genome Atlas Research Network, Van Allen EM, Cherniack AD, Ciriello G, Sander C, Schultz N. Oncogenic SignalingPathways in The Cancer Genome Atlas Cell 2018 ;173(2):321-337.e10.

[20] Adzhubei I, Jordan DM, Sunyaev SR. Predicting functional effect of human missense mutations using PolyPhen-2. Curr Protoc Hum Genet 2013; Chapter7:Unit7.20.

[21] Bromberg $Y$, Rost B. SNAP: predict effect of nonsynonymous polymorphisms on function. Nucleic Acids Res 2007;35(11):3823-35.

[22] Tate JG, Bamford S, Jubb HC, Sondka Z, Beare DM, Bindal $\mathrm{N}$, Boutselakis $\mathrm{H}$, Cole CG, Creatore C, Dawson E, Fish P, Harsha B, Hathaway C, Jupe SC, Kok CY, Noble K, Ponting L, Ramshaw CC, Rye CE, Speedy HE, Stefancsik R, Thompson SL, Wang S, Ward S, Campbell PJ, Forbes SA. COSMIC: the Catalogue Of Somatic Mutations In Cancer. Nucleic Acids Res 2019;47(D1):D941-D947.

[23] Tang Z, Li C, Kang B, Gao G, Li C, Zhang Z. GEPIA: a web server for cancer and normal gene expression profiling and interactive analyses. Nucleic Acids Res. 2017;45(W1):W98-W102.

[24] Jing X, Yang F, Shao C, Wei K, Xie M, Shen H, Shu Y. Role of hypoxia in cancer therapy by regulating the tumor microenvironment. Mol Cancer. 2019;18(1):157.

[25] Tan Z, Xu J, Zhang B, Shi S, Yu X, Liang C. Hypoxia: a barricade to conquer the pancreatic cancer. Cell Mol Life Sci. 2020;6.

[26] Leppänen J, Helminen O, Huhta H, Kauppila JH, Isohookana J, Haapasaari KM, Parkkila S, Saarnio J, Lehenkari PP, Karttunen TJ. Weak HIF-1alpha expression indicates poor prognosis in resectable pancreatic ductal adenocarcinoma. World J Surg Oncol 2018;16(1):127..

[27] Hoffmann AC, Mori R, Vallbohmer D, Brabender J, Klein E, Drebber U, Baldus SE, Cooc J, Azuma M, Metzger R, Hoelscher AH, Danenberg KD, Prenzel KL, Danenberg PV. High expression of HIF-1 $\alpha$ is a predictor of clinical outcome in patients with pancreatic ductal adenocarcinomas and correlated to PDGFA, VEGF, and bFGF. Neoplasia 2008;10(7):674-9. 